\title{
Fairness in Multi-hop Wireless Backhaul Networks: A Dynamic Estimation Approach
}

\author{
Jeng Farn Lee and YuanNeng Hsu \\ Department of Computer Science and Information Engineering, National Chung Cheng University, Taiwan \\ E-mail:jflee@cs.ccu.edu.tw
}

\begin{abstract}
In this work, we consider the problem of fairness for Transit Access Points (TAP) in multi-hop wireless backhaul networks. Existing approaches are not practical due to the requirement for modifications to the MAC layer or queueing operations of TAPs, or it is difficult to measure the effective link capacities between TAPs since this capacity must account for such factors as the MAC layer overhead, the effect of wireless interference, the hidden terminal problem, and multi-rate multi-channel issues. Moreover, some approaches are only suitable for TDMA-based MAC protocols. Therefore, we propose an effective and practical approach with two algorithms to enforce fairness in wireless backhaul networks. We also evaluate the performance of proposed approach via ns-2 simulations.
\end{abstract}

Keywords: fairness, wireless backhaul networks ${ }^{1}$

\section{INTRODUCTION}

IEEE 802.11-based wireless network is a promising wireless technology for accessing the Internet due to the characteristics of low cost, robustness, and ease of deployment. Recently, many studies have extended its applications from the traditional one-hop network access [1-4] to multi-hop communications, such as ad hoc networks, mesh networks, and backhaul networks [5-8]. In a multi-hop wireless backhaul network, traffic from mobile users to the wired Internet, and vice versa, is processed through multiple wireless Transit Access Points (TAPs) via a gateway, as illustrated in Fig. 1. TAPs may be located in different independent entities, such as restaurants, small business offices, private residences, or hot spots. However, under the Distributed Coordination Function (DCF) in 802.11, users located more hops away from the gateway suffer from low throughput, and in some cases, even starvation. The problem is caused by multi-hop relays, flow aggregation, and the underlying MAC layer mechanisms.

We demonstrate the unfair effects of TAPs in wireless backhaul networks via ns-2 simulations. Fig. 1 illustrates the simulation topology of a wireless backhaul network in which TAPs located two hops away are in the carrier sense range, but not the transmission range. The wireless link rate is set to $11 \mathrm{Mbps}$ and each traffic flow is generated as CBR UDP traffic with a fixed packet size of 1000 bytes, including the IP header. The MAC protocol used in these simulations is IEEE 802.11 DCF. The figure shows the simulation results with different traffic loads. We observe that (i) TAPs located more hops away from the gateway obtain less end-to-end throughput than those less hops away when the traffic load are more than the link capacity (i.e., the sending rate is more than $300 \mathrm{kbps}$ ), resulting in spatial bias in the network; (ii) the degree of bias increases when the offered rates

\footnotetext{
${ }^{1}$ Permission to make digital or hard copies of all or part of this work for personal or classroom use is granted without fee provided that copies are not made or distributed for profit or commercial advantage and that copies bear this notice and the full citation on the first page. To copy otherwise, to republish, to post on servers or to redistribute to lists, requires prior specific permission and/or a fee.
} QShine 2008, July 28-31, 2008, Hong Kong, Hong Kong.

Copyright 2008 ICST ISBN 978-963-9799-26-4 DOI 10.4108/ICST.QSHINE2008.3837 of TAPs increase because both transit and local data are put into the same queue, thereby increasing the frame dropping rates of the transit data from other TAPs when the arrival rates of mobile users increase; and (iii) although $\mathrm{TAP}_{7}$ and $\mathrm{TAP}_{2}$ are two hops away from the gateway, $\mathrm{TAP}_{2}$ achieves less end-to-end throughput because more neighbors contend for the wireless channel.

The above effects call for a fair bandwidth allocation mechanism for wireless backhaul networks. Existing approaches are not practical due to the requirement for modifications to the MAC layer or queueing operations of TAPs, or it is difficult to measure the effective link capacities between TAPs since this capacity must account for such factors as the MAC layer overhead, the effect of wireless interference, the hidden terminal problem, and multi-rate multi-channel issues. Moreover, some approaches are only suitable for TDMA-based wireless MAC protocols. In this paper, we evaluate existing fairness mechanisms and propose an effective and practical approach as well as two algorithms to enforce fairness in wireless backhaul networks. We also evaluate the performance of proposed approach via ns-2 simulations.

The remainder of the paper is organized as follows. In Section II, we discuss the fairness model suitable for wireless backhaul networks and review related works on fairness mechanisms. In Section III, we present our proposed fairness mechanism. The performance of the proposed approach is evaluated via simulations based on ns- 2 in Section IV. Our conclusions and future research directions are detailed in Section V.

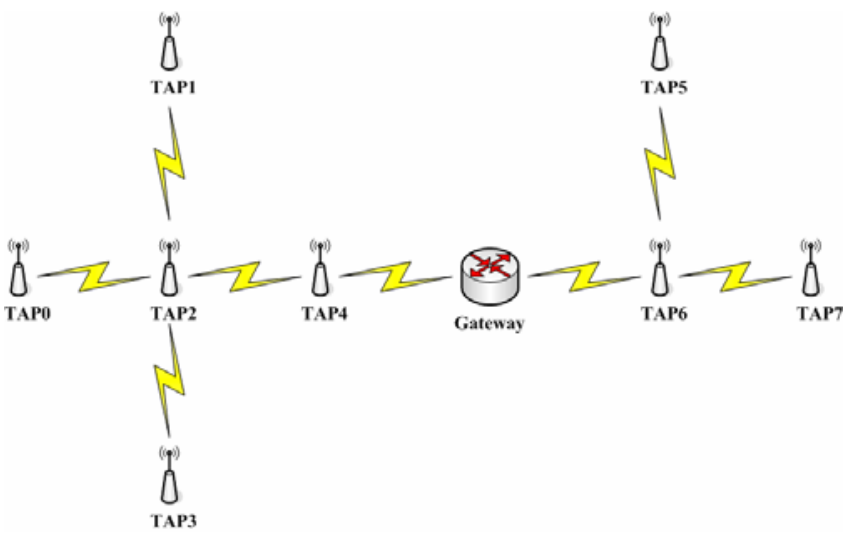

Figure 1: A multi-hop wireless backhaul network 


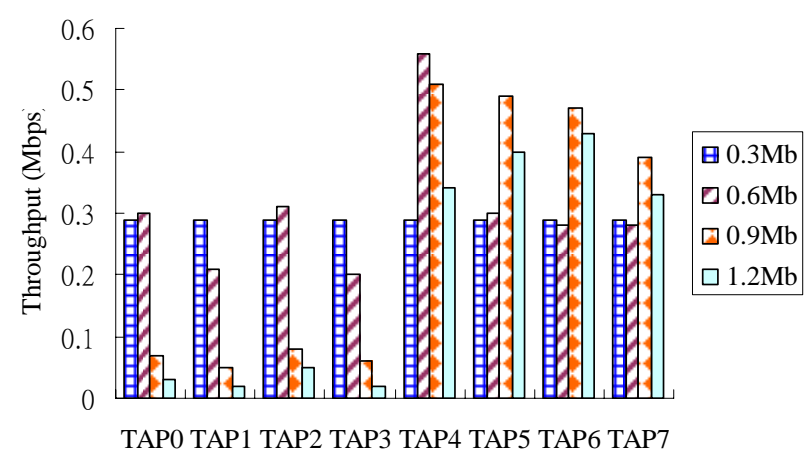

Fig. 2. Throughput performance of TAPs

\section{RELATED WORK}

In this section, we consider the fairness reference model and existing fairness mechanisms in multi-hop wireless backhaul networks. These fairness mechanisms can provide fairness among TAPs; however, they all have some drawbacks in implementation issues, high packet loss rates, high operation costs, or limitations on network topology and the applied MAC protocols. We will review and classify these fairness mechanisms according to their design and controlling factors.

\subsection{FAIRNESS REFERENCE MODEL}

The fairness reference model proposed by Gambiroza et al. [9] is very adaptive for multi-hop wireless backhaul networks. The model is based on four constraints. First, the granularity of fairness is a TAP-aggregated flow. The egress traffic of each TAP should be treated as a single aggregate flow, independent of the number of local micro-flows or mobile devices supported by the TAP. Second, maximal spatial reuse must be ensured. Third, to avoid the IEEE 802.11 performance anomaly reported in [10], the model uses air time rather than throughput as the network resource to be shared fairly. Specifically, throughput-based allocation leads to serious performance degradation, since the station with the lowest channel quality determines the throughput achievable by all stations. Finally, spatial bias must be eliminated so that TAPs located farther away from the gateway will not receive disproportionately less air time than nodes close to the gateway. This property is essential in the deployment of multi-hop wireless backhaul architectures. In other words, TAPs in different locations should not be penalized because the network's performance is distance-dependent. Note that the fairness reference model described above is used to define fairness for TAPs destined for the same gateway. It is impractical to define fairness among TAPs destined for different gateways because each gateway may be associated with its own unique network topology, wireless resource, contention, and other resource constraints. Therefore, we focus on the fairness mechanism for TAPs in a backhaul network with only one gateway. Networks with more gateways can be considered as multiple backhaul networks, each with a single gateway.

To determine the effects of each fairness constraint on the capacity of a multi-hop wireless backhaul network, Gambiroza et al. propose a model that computes the target end-to-end throughput of each TAP with different fairness objectives. However, they focus on backhaul networks with no spatial reuse, i.e., only one link can be active at any given time, which is typical of networks where all links mutually contend for bandwidth. In contrast, the authors of [11] provide a general formulation for obtaining target throughputs of TAPs under fairness constraints in a more general backhaul network, where spatial reuse is possible and some TAPs may not always be backlogged.

\subsection{EXISTING FAIR MECHANISMS}

The fairness mechanisms in [9] and [12] control the sending rates of TAPs being their target throughputs, which are the desired end-to-end throughputs based on the four fairness constraints in the proposed fairness reference model. Therefore, each TAP needs to measure the offered load of its local traffic, i.e., the arrival rate of aggregate traffic from all local mobile users during a predefined measurement time period, and the available capacity of each link connecting to an adjacent TAP. The offered load and the capacity of each link incident to each TAP are then exchanged between TAPs periodically. As a result, the end-to-end throughput for each TAP-aggregated flow with fairness constraints can be computed as a function of the offered load of each TAP. The advantage of controlling TAPs' sending rates is that it does not need to modify the queueing, forwarding and contention operations of TAPs because the finely-tuned sending rates eliminate contention behavior in the network. However, the measured capacity must consider factors like the MAC layer overhead, the effect of wireless interference, the hidden terminal problem, and multi-rate multi-channel issues. As a result, it is very difficult to measure the capacity in real networks. Moreover, the information exchanged between TAPs increases the load of wireless backhaul networks.

In contrast, the approaches proposed in [13] and [14] modify the queuing, forwarding and contention behavior of TAPs to enforce the fairness model. The authors of [13] evaluate the fairness and throughput performance of various queuing schemes, and show that per-flow queuing (i.e., one queue for each TAP-aggregate flow) at intermediate TAPs is required to achieve fairness. Moreover, to ensure throughput efficiency, TAPs that need to transmit more data should be allocated extra bandwidth via MAC-layered QoS mechanisms. However, since they do not control the sending rates of TAPs, more and more transit data will be dropped by TAPs closer to the gateway. Therefore, the bandwidth used by previous TAPs to transmit and relay those dropped frames will be wasted. In addition, per-flow queuing requires more hardware and processing budget. Meanwhile, under the mechanism proposed in [14], there are only two queues at intermediate TAPs: one for local data and the other for transit data. Liu and Liao study how channel access behavior and the forwarding probability of the transit queue of each TAP influence network performance, and then derive the throughput and packet delay experienced by TAPs at different distances (hop counts) from the gateway. However, they assume each $\mathrm{x}$-hop TAP must be a relay for the same number of $(x+1)$-hop TAPs (e.g. Fig. 3) such that the applied network topologies are limited.

Another way to achieve fairness is to schedule the transmitting links at each time slot in a wireless backhaul network. For example, Fig. 5 shows a possible upstream scheduling scenario with spatial reuse that guarantees the TAP-aggregate fairness (i.e., from TAP1 to TAP10) in the backhaul network shown in Fig. 4. This approach has been proved notoriously difficult to realize for two reasons: (i) even if global information, such as the network topology and link capacities, is available, the scheduling problem of finding the optimal link transmission set at each time slot is NP-complete; and (ii) the link 
schedule needs to be re-calculated when the network topology or the traffic loads of mobile clients change. Although [15] and [16] propose low-complexity distributed mechanisms, the approaches can only be used in wireless networks that adopt TDMA-based MAC protocols like IEEE 802.16.

To overcome the limitations or drawbacks of the above mechanisms, we propose a practical approach for achieving fairness in wireless backhaul networks. The basic idea is to control the sending rates of TAPs to their target throughputs under the fairness constraints since this approach does not need to modify the MAC layer and queuing operations of TAPs. However, unlike the approach in [9] and [12], we do not measure the effective link capacities of TAPs and then calculate the target throughputs because it is difficult to measure the link capacities effectively in real networks. We use an adapted approach and propose two algorithms to estimate the target throughput of each TAP in the backhaul network with high accuracy.

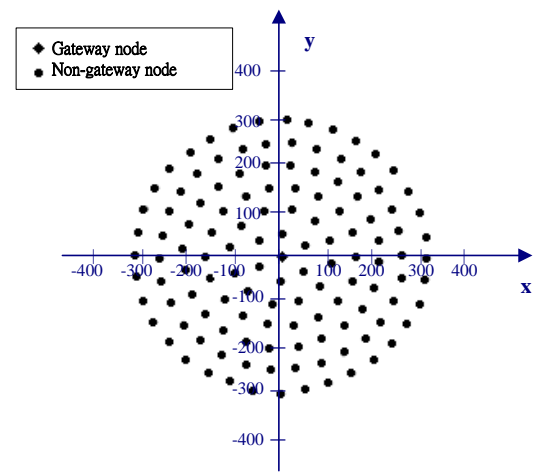

Figure 3. A 126-node network

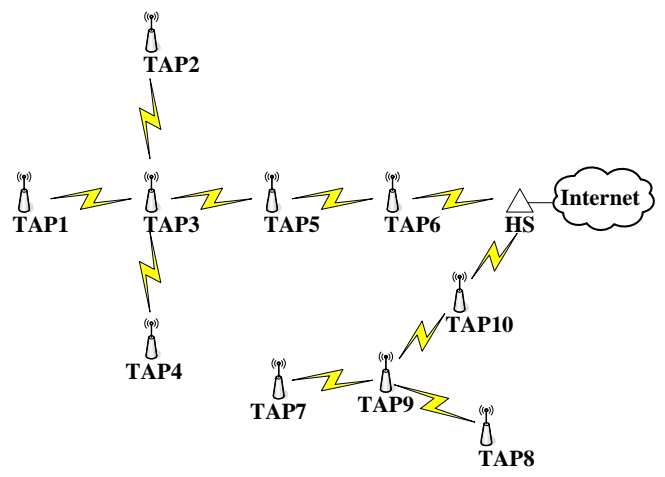

Figure 4. A wireless backhaul network

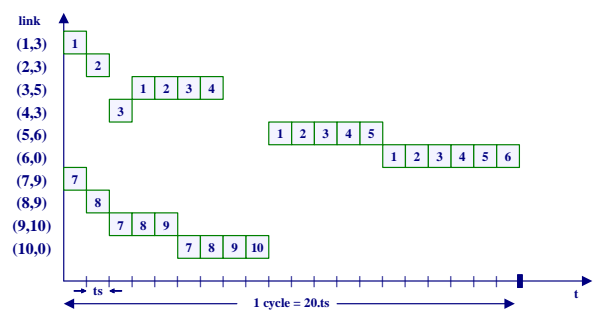

Figure 5. A possible TDMA scheduling scenario with spatial reuse for the upstream links in the backhaul network in Fig. 4. In this example, $\mathrm{T}=20$.

\section{TAP-Aggregate Fairness Mechanisms}

In our work, we consider static, cooperative TAPs in wireless multi-hop backhaul networks. We assume that data will not be exchanged between TAPs, and data sent from one TAP will not be split among different gateways at intermediate TAPs. All TAPs are always backlogged and have the same link capacity as adjacent TAPs. However, our mechanisms can easily be extended to backhaul networks in which the TAPs have different link capacities and not all TAPs are saturated. We assume the gateway can 1) measures the average throughput of each TAP-aggregate flow for the previous measurement period; 2) calculates the sending rate of each TAP in the next time period based on our algorithm; and 3) distributes this information to TAPs at the beginning of the next time period. If the TAPs find the target sending rates, the gateway stops the above operations. Here, the 'target sending rate' means the corresponding target throughput of TAPs based on the fairness constraints in the backhaul networks. Since we assume that the link capacities are the same, the target sending rates of TAPs should have the same value. If the operations of gateways are difficult to modify, each TAP can calculate the average throughput in previous time period, and distribute the information to other TAPs in a similar way to that in Open Shortest Path First (OSPF) protocol. Then, based on our algorithm, each TAP can calculate its sending rate for this time period based on the average throughput of other TAPs.

We use two simple algorithms, called Binary Search based on Sending Rates (BSSR) and Max-Min Search (MMS), to estimate the proper sending rates of TAPs in this round based on the average throughput of TAPs in the previous round. In both algorithms, the initial sending rate of each TAP is $500 \mathrm{kbps}$, but the rate is increased by $\alpha$ kbps if the TAP can achieve a throughput similar to its sending rate in the initial state. We will investigate the values of initial sending rate and $\alpha$ in a future work. If there is unfairness between the throughputs of TAPs, the estimation process goes into the adaptation state, and MMS and BSSR use different strategies to decide the new sending rate for the next round. We assume that each TAP has the same throughput (i.e., it is fair between all TAPs) if the difference ratio between the maximum and minimum throughput of the current round is less then $\beta$ of the maximum throughput. In this work, we nominate a small value, such as $3 \%$, in order to demonstrate that our algorithms achieve high accuracy and converge rapidly. Moreover, both algorithms terminate if the difference between two continuous sending rates is less than $\gamma$ of the previous sending rate. 


\subsection{Binary Search based on Sending Rates (BSSR)}

The BSSR algorithm is an intuitive method that tries to estimate the sending rate for a backhaul network by a binary search of the sending rates in previous rounds. Let $\min r$ and $\max r$ denote, respectively, the minimum and maximum throughput of TAPs in the previous round. In addition, we use low and high to denote the lower and upper bounds of the new sending rate in the binary search process. The initial sending rate is $500 \mathrm{kbps}$, and is increased by $\alpha$ if there is no unfairness between TAPs (i.e., $\max \_r-\min \_r>\beta * \max \_r$ ) because the backhaul network can afford to transmit more traffic; otherwise, BSSR goes into the adaptation state. If there is unfairness between TAPs in the adaptation state, the old sending rate should be greater than the target value, so BSSR sets the previous sending rate as high; otherwise, it is set as low. Consequently, BSSR selects the mean of the low and high as the new sending rate. Fig. 6 details the BSSR algorithm. Although BSSR can eventually find the approximate target sending rate of TAPs for the fairness reference model, it may take several rounds to converge. Moreover, the sending rates of TAPs may fluctuate a great deal in different rounds since the sending rates may increase or decrease in the adaptation state.

\subsection{Max-Min Search (MMS)}

To speed up the convergence time of the search process and provide stable sending rates for TAPs in different rounds, we propose the Max-Min Search (MMS) algorithm. The initial state of MMS works the same as that in BSSR algorithm. However, the MMS takes the mean value of $\min r$ and $\max r$ as the new sending rate based on the concept that the target sending rate should be between the maximum and minimum throughput if there is unfairness between TAPs in the previous round. Fig. 7 shows the MMS algorithm. Note that, in the adaptation state, the new sending rate will always be lower than the old sending rate because the value of new sending rate will be between max_r and $\min _{-} r$, and $\max _{-} r$ must be less than the old sending rate.

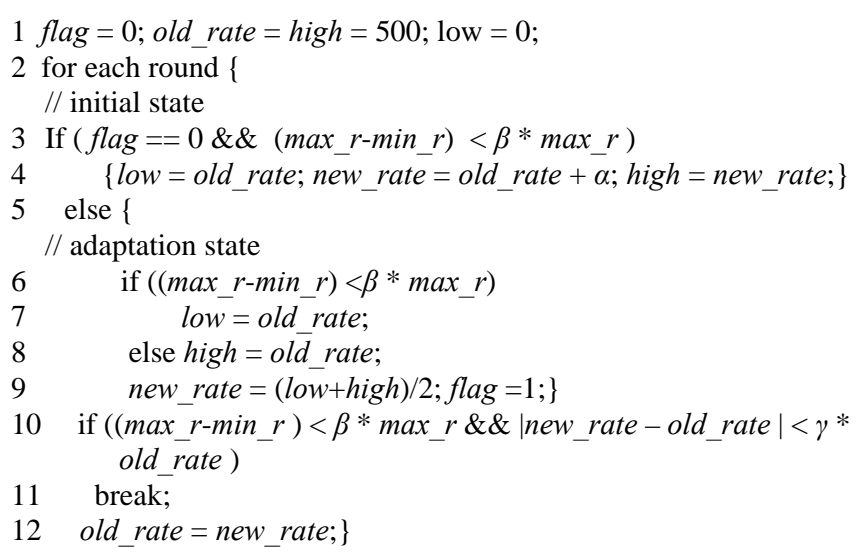

Figure 6. The BSSR algorithm

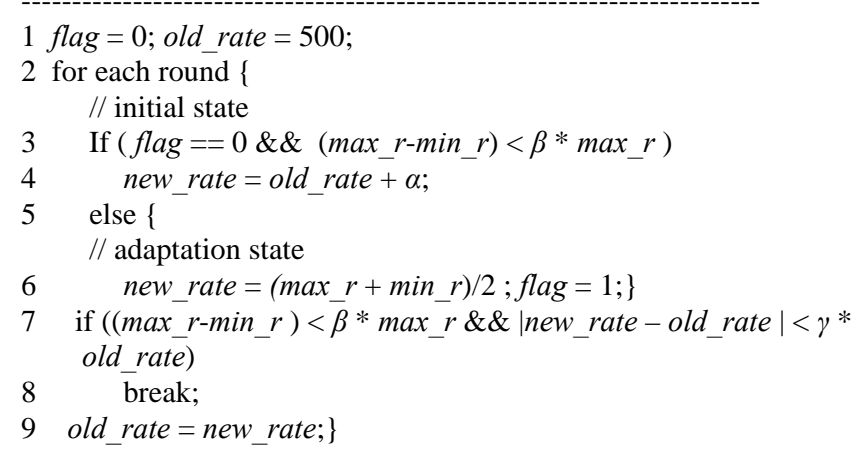

Figure 7. The MMS algorithm

\section{Performance Evaluation}

In this section, we evaluate the performance of the proposed algorithms via ns-2 simulations. The wireless link rate is set to $11 \mathrm{Mbps}$ and each traffic flow is generated as CBR UDP traffic with a fixed packet size of 1000 bytes, including the IP header. The MAC protocol used in the simulations is IEEE 802.11 DCF without RTS/CTS; the duration of each round is one second; and the values of $\beta$ and $\gamma$ are both $3 \%$.

We compare the performance of MMS and BSSR under three simulation scenarios: (i) the "Parking Lot" wireless backhaul network scenario (Fig. 8(a)); (ii) a network with 8 TAPs and one gateway, which is located on the edge of the topology (Fig. 9(a)); and (iii) a network with 8 TAPs, but the gateway is located in the center of the topology (Fig. 10(a)). Figs. 8 to 10 plot the average throughput performance of TAPs for BSSR and MMS (the (b) sub-figures are for BSSR and the (c) sub-figures are for MMS). Tables 1 and 2 show the sending rates of BSSR and MMS for the "Parking Lot" scenario. Due to space limitations, we do not show the sending rates in detail for scenarios (ii) and (iii).

In the second round in Table1, the difference between $\max r$ and $\min r$ (i.e., 976-867) is more than 3\% ( $\beta$ ) of $\max r$ such that BSSR goes into the adaptation state, and the new sending rate of TAPs in the next round is the mean of low and high (i.e., $(500+1000) / 2)$. In the third round, because the difference in the maximal and minimal average throughput is less than $3 \%$, which means the network can deal with more data traffic, the BSSR algorithm sets the previous sending rate (i.e., $750 \mathrm{kbps}$ ) as low, as shown in line 7 of Fig. 6. Consequently, the new sending rate of round 4 is $875 \mathrm{kbps}$. However, in round 5, BSSR sets the previous sending rate as high because there is unfairness between the throughput of the TAPs; thus, the sending rate in round 6 is less than that in round 5.

Table 2 shows sending rates of TAPs in scenario (i) for MMS in different rounds. In the third round, the difference between the maximal and minimal average throughput is less than $3 \%$, which means all TAPs have the same throughput, and the difference between the new and old sending rates is also less than $3 \%(\gamma)$; therefore, the MMS algorithm terminates, as shown in line 7 of Fig. 7. From the results in Tables 1 and 2, we observe that BSSR needs more rounds to achieve convergence and there are more fluctuations between the sending rates of different rounds in BSSR.

Sub-figures b and c of Figs. 8 to 10 show the simulation results for the three scenarios. The target sending rates of the three scenarios are 
913kbps, 232kbps and 323kbps, respectively. We observe that both algorithms accurately estimate the target sending rates for all scenarios. Specifically, MMS only needs about three rounds to estimate the target sending rates of TAPs in a wireless backhaul network. Since its sending rates in the adaptation state are stable and it converges rapidly, MMS is also suitable for backhaul networks with dynamic traffic loads, dynamic or mobile TAPs. Compared to MMS, BSSR converges more slowly and there are more fluctuations between the sending rates of different rounds.

Table 1 . The sending rates of BSSR for scenario 1

round sending TAP 0 TAP 1 TAP next sending rate

\begin{tabular}{|c|c|c|c|c|c|}
\hline & rate & & & 2 & \\
\hline 1 & 500 & 492 & 484 & 484 & $500+500=1000$ \\
\hline 2 & 1000 & 867 & 859 & 976 & $(500+1000) / 2=750$ \\
\hline 3 & 750 & 726 & 734 & 734 & $(750+1000) / 2=875$ \\
\hline 4 & 875 & 851 & 859 & 851 & $(875+1000) / 2=938$ \\
\hline 5 & 938 & 882 & 890 & 914 & $(938+875) / 2=906$ \\
\hline 6 & 906 & 890 & 890 & 890 & $(906+938) / 2=922$ \\
\hline 7 & 922 & & & & \\
\hline
\end{tabular}

Table 2. The sending rates of MMS for scenario 1

$\begin{array}{cccccc}\text { round } & \begin{array}{c}\text { sending } \\ \text { rate }\end{array} & \text { TAP0 } & \text { TAP1 } & \text { TAP2 } & \text { next sending rate } \\ 1 & 500 & 492 & 484 & 484 & 500+500=1000 \\ 2 & 1000 & 867 & 859 & 976 & (976+859) / 2=918 \\ 3 & 918 & 906 & 890 & 898 & (906+890) / 2=898\end{array}$

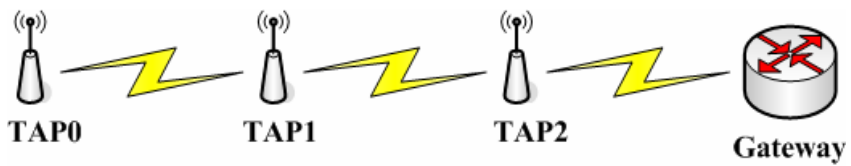

Figure 8-a.Scenario 1: The Parking Lot Scenario

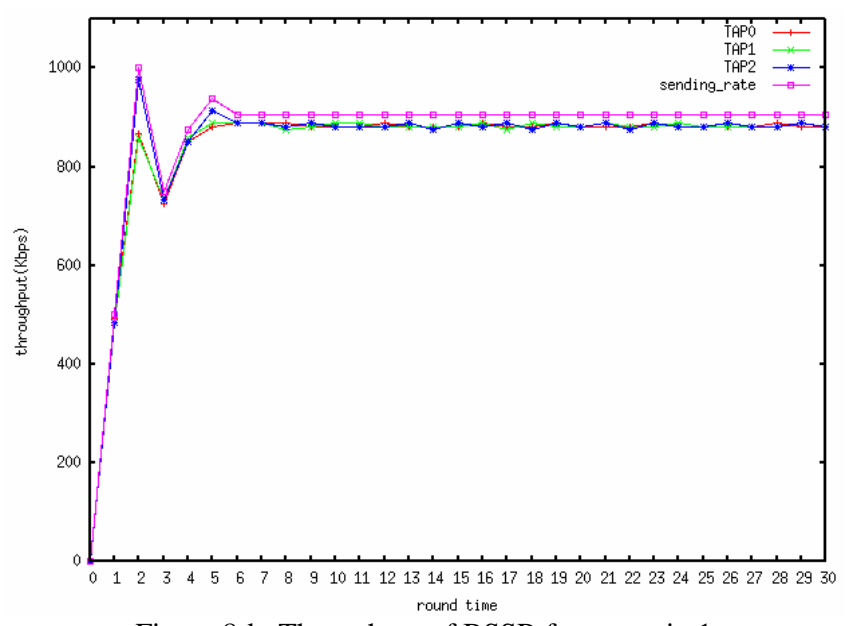

Figure 8-b. Throughput of BSSR for scenario 1

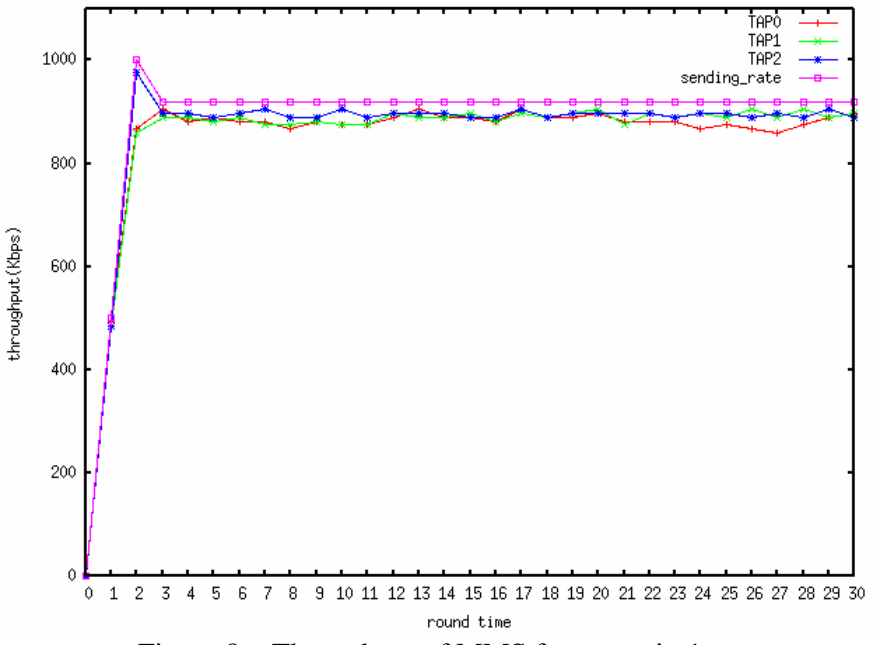

Figure 8-c Throughput of MMS for scenario 1

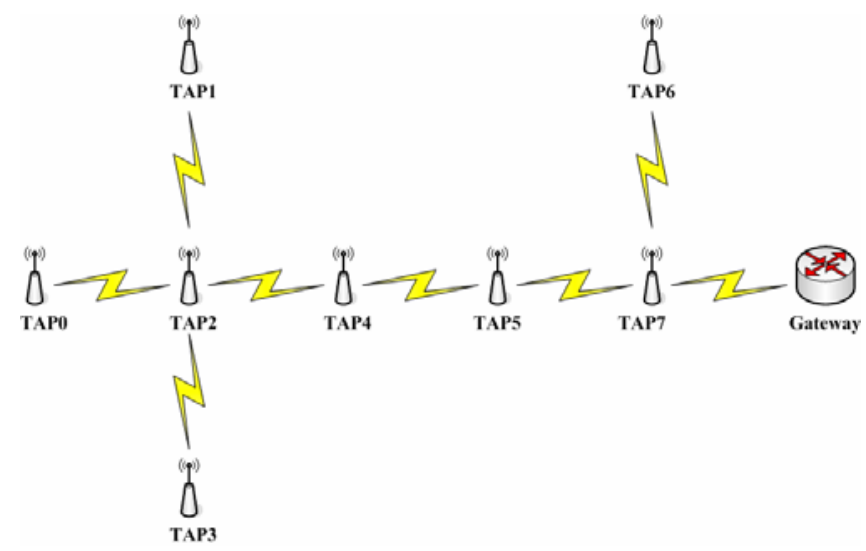

Figure 9-a. Scenario 2: eight TAPs with the gateway located at the border

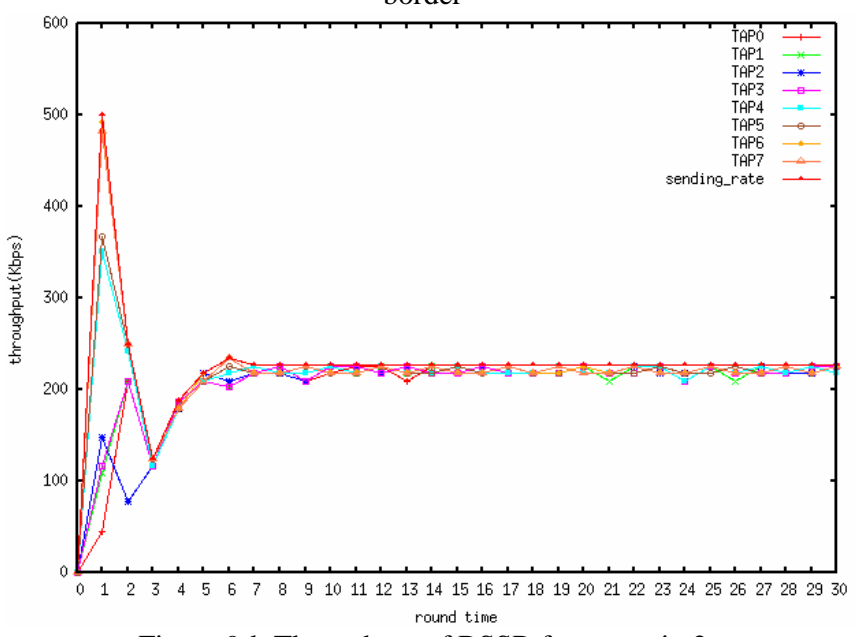

Figure 9-b Throughput of BSSR for scenario 2 


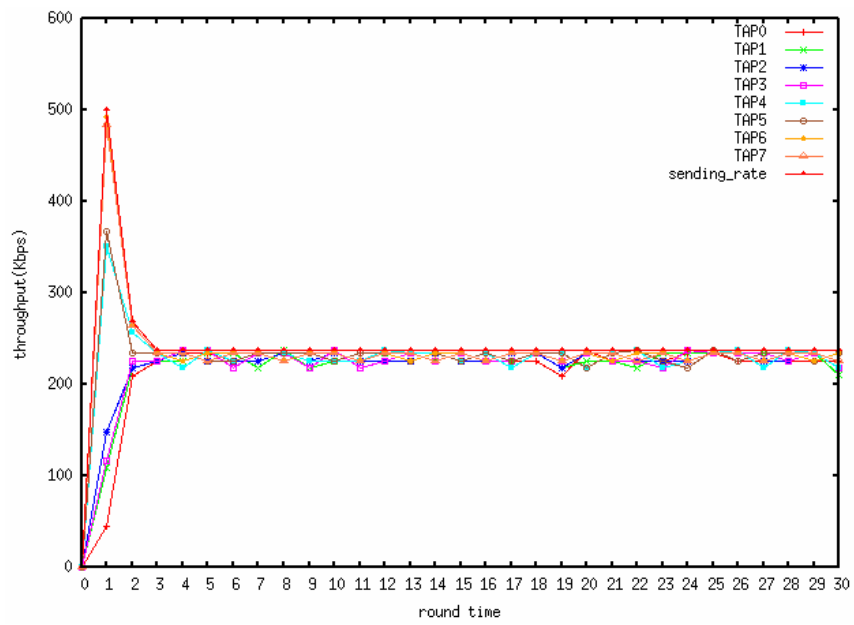

Figure 9-c Throughput of MMS for scenario 2

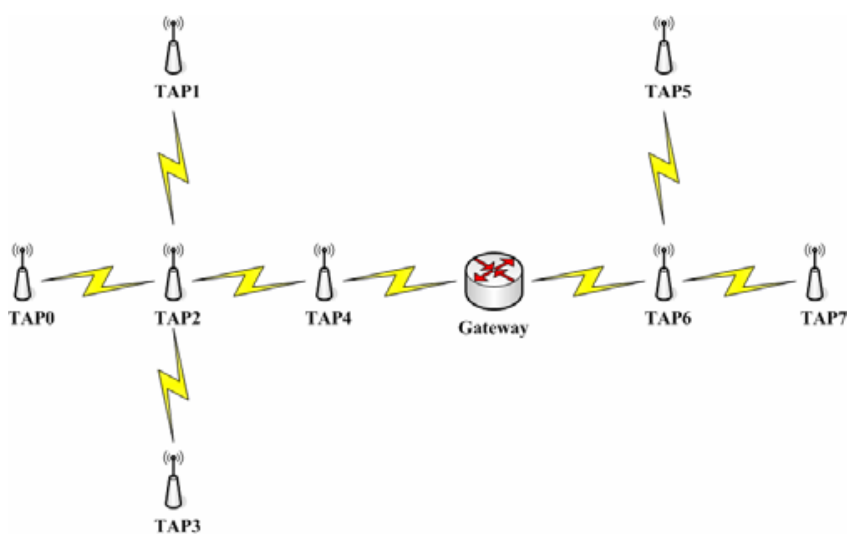

Figure 10-a .Scenario 3: eight TAPs with the gateway located in the center

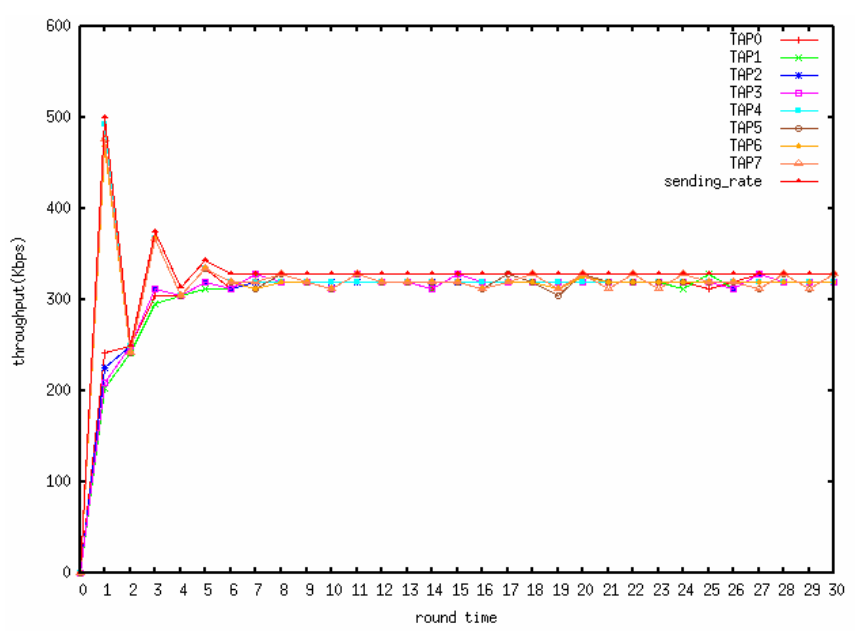

Figure 10-b. Throughput of BSSR for scenario 3

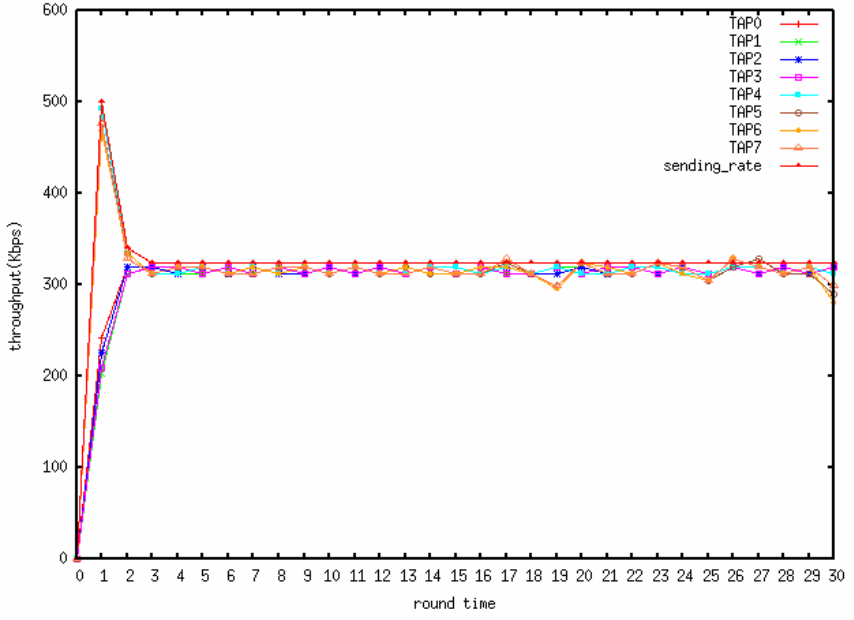

Figure 10-c. Throughput of MMS for scenario 3

\section{CONCLUSION AND FUTURE WORK}

In this work, we address the problem of fairness between TAPs in multi-hop wireless backhaul networks. We propose an effective and practical approach and two algorithms, BSSR and MMS, to enforce the fairness condition in wireless backhaul networks. Specifically, we control the sending rates of TAPs to their target throughputs under the fairness constraints because, under this approach, we do not need to modify the MAC layer and queuing operations of the TAPs. The algorithms estimate the target throughput of each TAP efficiently, instead of measuring the link capacities of TAPs. Performance evaluations based on ns-2 simulations show that both BSSR and MMS can accurately estimate the target sending rates of TAPs. Moreover, since MMS converges rapidly and its sending rates in different rounds are stable, it is also suitable for backhaul networks with dynamic traffic loads, dynamic TAPs or mobile TAPs.

In the future, we will consider backhaul networks with different link capacities, and scenarios where TAPs may communicate with each other and they do not always have data to send. Moreover, we will try to obtain good estimated values for the initial sending rates and $\alpha$ for different network topologies.

\section{ACKNOWLEDGEMENTS}

This work was supported in part by National Science Council, Taiwan, under Grant Number NSC 96-2218-E-194-004.

\section{REFERENCES}

[1] W. Pattara Atikom, P. Krishnamurthy, and S. Banerjee, "Distributed Mechanisms for Quality of Service in Wireless LANs," IEEE Wireless Communications, Vol. 10, Issue 3, page(s): 26-34, June 2003

[2] Jeng Farn Lee, Wanjiun Liao, and Meng Chang Chen, "Inter-Frame Space (IFS) Based Distributed Fair Queuing in IEEE 802.11 WLANs,” IEEE Transactions on Vehicular Technology, page(s): 1366-1373 Vol. 56, NO. 3, Mar. 2007

[3] Jeng Farn Lee, Wanjiun Liao, and Meng Chang Chen, "A Differentiated Service Model for the Enhanced Distributed Channel Access (EDCA) of IEEE 802.11e WLANs," 
ACM/Springer Mobile Networks and Applications (MONET) Special Issue on Wireless Broadband Access, page(s): 69-77 Vol. 12, No. 1, Feb. 2007

[4] Daji Qiao and Kang G. Shin, “Achieving Efficient Channel Utilization and Weighted Fairness for Data Communications in IEEE 802.11 WALN under the DCF," In Proc. of ACM IWQoS, page(s): 227-236, 2002

[5] K. Sundaresan, H. Y. Hsieh and R. Sivakumar, "IEEE 802.11 over multi-hop wireless networks: problems and new perspectives,” In Ad Hoc Networks, page(s): 109-132, 2004

[6] X. Wang and K. Kar, "Cross-Layer Rate Control for End-to-End Proportional Fairness in Wireless Networks with Random Access," In Proc. of ACM MobiHoc, page(s): 157-168, 2005

[7] J. Robinson and E. Knightly, "A Performance Study of Deployment Factors in Wireless Mesh Networks," in Proceedings of IEEE INFOCOM 2007, Anchorage, AK, May 2007

[8] J. Shi, O. Gurewitz, V. Mancuso, J. Camp, and E. Knightly, "Measurement and Modeling of the Origins of Starvation in Congestion Controlled Mesh Networks," in Proceedings of IEEE INFOCOM 2008, Phoenix, AZ, April 2008.

[9] V. Gambiroza, B. Sadeghi, and E.W. Knightly, "End-to-end performance and fairness in multihop wireless backhaul networks," In Proc. of ACM MobiCom, page(s): 287-301, 2004

[10] M. Heusse, F. Rousseau, G. Berger-Sabbatel, and A. Duda. “Performance Anomaly of 802.11b," In Proc. of IEEE Infocom, page(s):836-843, 2003

[11] Jeng-Farn Lee, Wanjiun Liao, and Meng-Chang Chen, “An Incentive-based Fairness Mechanism for Multi-hop Wireless Backhaul Networks with Selfish Nodes," IEEE Transactions on Wireless Communications, Vol. 7, No. 2, Feb. 2008

[12] J. Camp J. Robinson, C. Steger, and E. Knightly, "Measurement Driven Deployment of a Two-Tier Urban Mesh Access Network," in Proceedings of ACM MobiSys , Uppsala, Sweden, June 2006

[13] J. Jun and Sichitiu, M.L., "Fairness and QoS in multihop wireless networks,” In Proc. of IEEE VTC, page(s): 2936-2940, 2003

[14] Tehuang Liu and Wanjiun Liao, "Location-Dependent Throughput and Delay in Wireless Mesh Networks," IEEE Transactions on Vehicular Technology, Vol. 57, No. 2, Mar. 2008

[15] N. Ben Salem and J. Hubaux, "A Fair Scheduling for Wireless Mesh Networks,," in Proceedings of WiMesh 2005, September 2005.

[16] A. Kabbani, T. Salonidis, and E. Knightly, "A Distributed Low-Complexity Maximum-Throughput Scheduler for Wireless Backhaul Networks," in Proceedings of IEEE INFOCOM 2007, Anchorage, AK, May 2007. 